\title{
The Clinical Significance of Interleukin-6, C-reactive Protein, and Procalcitonin in the Early Recognition of Nosocomial Infections in Preterm Infants
}

\section{Xuewei Cui}

Shengjing Hospital of China Medical University https://orcid.org/0000-0001-5181-8740

\section{Yongyan Shi}

Shengjing Hospital of China Medical University

\section{Xinyi Zhao}

Shengjing Hospital of China Medical University

\section{Xindong Xue}

Shengjing Hospital of China Medical University

Jianhua Fu ( $\square$ liying58522@163.com )

Department of Pediatrics, Shengjing Hospital of China Medical University, Shenyang, China 6 Correspondence to: Jianhua Fu, Department of Pediatrics, Shengjing Hospital of China https://orcid.org/0000-0003-0660-4211

\section{Research article}

Keywords: C-reactive protein, Interleukin-6, Nosocomial infection, Preterm infants, Procalcitonin

Posted Date: November 23rd, 2020

DOl: https://doi.org/10.21203/rs.3.rs-106968/v1

License: (9) This work is licensed under a Creative Commons Attribution 4.0 International License. Read Full License 
1 The clinical significance of interleukin-6, C-reactive protein, and procalcitonin in the early recognition of nosocomial infections in preterm infants

3 Short title: IL-6, CRP, and PCT for neonatal sepsis Xuewei Cui, Yongyan Shi, Xinyi Zhao, Xindong Xue and Jianhua Fu* Department of Pediatrics, Shengjing Hospital of China Medical University, Shenyang, China

6 Correspondence to: Jianhua Fu, Department of Pediatrics, Shengjing Hospital of China

$7 \quad$ Medical University, Shenyang, China. E-mail: liying58522@163.com, Phone

8 number:+(86)18940251830.

9 


\section{Abstract}

11 Background: This study assessed the clinical value of interleukin-6 (IL-6), C-reactive

12 protein (CRP), and procalcitonin (PCT) for early recognition of nosocomial infections in

13 preterm infants.

14 Methods: 120 neonates were enrolled, 89 with sepsis were compared with 31 noninfected controls. IL-6, CRP and PCT were measured at certain stages of infection. Receiver-operating

16 characteristic (ROC) curve analysis was used to determine the best cutoff values of IL-6, CRP, 17 and PCT for diagnosing sepsis.

18 Results: The levels of IL-6, CRP, and PCT in the sepsis group were significantly higher than

19 those in the control group before antibiotic treatment. In the sepsis group, IL-6 peaked 6

20 hours after the infection onset and dropped to normal 24-48 hours after the infection was

21 controlled. The best cutoff values of IL-6, CRP, and PCT were $>99.6 \mathrm{pg} / \mathrm{mL},>9.27 \mathrm{mg} / \mathrm{L}$, and $>2.33 \mathrm{ng} / \mathrm{mL}$, respectively. The areas under the ROC curves were $0.888,0.823$, and 0.953 , respectively. When PCT was combined with IL-6 or IL-6 and CRP, the sensitivity and specificity were $>85 \%$.

Conclusion: IL-6, CRP, and PCT are reliable indicators for early diagnosis of nosocomial infections in preterm infants. Combining PCT and IL-6 CRP, IL-6, and PCT levels could improve the accuracy of clinical diagnosis.

Keywords: C-reactive protein; Interleukin-6; Nosocomial infection; Preterm infants;

Procalcitonin

\section{Key Notes}

31 Neonatal sepsis has an insidious onset and progresses rapidly. IL-6, CRP, and PCT are 
32 reliable indicators for early diagnosis of nosocomial infections in preterm infants. The best

33 cutoff values of IL-6, CRP, and PCT were $>99.6 \mathrm{pg} / \mathrm{mL},>9.27 \mathrm{mg} / \mathrm{L}$, and $>2.33 \mathrm{ng} / \mathrm{mL}$,

34 respectively. Combining PCT and IL-6 CRP, IL-6, and PCT levels could improve the

35 accuracy of clinical diagnosis. Monitoring these levels is fast, convenient, and worthy of

36 promotion worthwhile. 


\section{Background}

Neonatal sepsis is a systemic infectious disease caused by pathogens such as bacteria, fungi, and viruses that invade the circulation, grow and multiply, and produce toxins within 28 days after birth. According to the onset time (before or after 3 days of age), it can be divided into early-onset sepsis (EOS) and late-onset sepsis (LOS) [1]. Sepsis is the main cause of neonatal morbidity and death. The most common causes of neonatal sepsis include premature delivery, birth-related diseases, and infections. The incidence is $1-8 / 1,000$ live births. The lower the birth weight, the higher the incidence of neonatal sepsis. The incidence of neonatal sepsis in infants with very low birth weights may reach 164/1000, and the incidence of long-term hospitalization may reach 300/1000 [2]. The clinical signs of neonatal sepsis are atypical: It often manifests as respiratory distress, tachycardia or bradycardia, poor perfusion, feeding intolerance, jaundice, and abnormal body temperature. The nonspecific clinical signs increase the difficulty in diagnosing neonatal sepsis. Therefore, clinical diagnosis is an unreliable early indicator of sepsis [3].

The risk of developing sepsis is higher in neonates due to their immature immune systems, low leukocyte phagocytic activity, low cytokine production, poor humoral immunity, weak skin barrier, and the use of invasive procedures such as mechanical ventilation, central venous catheterization, and lumbar puncture [4, 5]. In 2013, the World Health Organization announced the results of an assessment of the causes of death of 2.8 million neonates worldwide. There were an estimated 420,000 neonatal deaths caused by sepsis per year, accounting for $15.0 \%$ of neonatal deaths. The incidence of neonatal sepsis has been gradually increasing worldwide, and the mortality rate was relatively high [6]. The onset of neonatal 
sepsis is relatively insidious. However, it progresses rapidly and is often accompanied by

60 severe complications, such as shock and multiple organ dysfunction. Therefore, early

61 diagnosis is essential to reducing the mortality of neonatal sepsis and improving the

62 prognosis. At present, the laboratory tests that are used for the diagnosis of sepsis include

63 bacterial culture, white blood cell counts, platelet counts, neutrophil percentages, cell surface

64 antigen and bacterial gene detection. However, because of unclear diagnostic thresholds,

65 complex detection methods, false-negative results, and other reasons, there is no ideal

66 indicator for the early diagnosis of neonatal sepsis. The gold standard for the diagnosis of

67 sepsis is blood culture, but the reporting of results may take $24-48$ hours. The accuracy of

clinical diagnosis is often affected by an insufficient blood sample, administration of

69 antibiotics before blood collection, and irregular operations. In addition, out of a fear of

missing a diagnosis of sepsis, which might result in shock and other serious complications,

physicians often administer broad-spectrum antibiotics empirically and adjust them after the

results of drug susceptibility tests are reported, which can lead to antibiotic abuse, the

presence of multidrug-resistant bacteria, fungal infections, and impaired intestinal immune

functioning [7]. Therefore, testing methods with good accuracy, high sensitivity and specificity, and quick results are urgently needed to guide the clinical diagnosis and treatment of neonatal sepsis. This study assessed the diagnostic accuracy of CRP, IL-6, PCT, singly and in combination, for the early diagnosis of nosocomial infections in preterm infants. 
Methods

\section{Participants}

This study was conducted among preterm infants admitted to the Department of Neonatology, Shengjing Hospital of China Medical University, from July 2019 to July 2020. The inclusion criteria included an age $>3$ days in preterm infants whose gestational age was $\geq 30$ and $\leq 34$ weeks at birth, with stable vital signs and hemodynamic parameters. The exclusion criteria included the mother's use of glucocorticoids before delivery; diagnosis with a community-acquired infection; incomplete clinical records; antibiotic use before the first blood collection; and a suspicion that the infant had inherited metabolic diseases, congenital malformations, prenatal or intrapartum asphyxia, or immunodeficiency.

This study was conducted in accordance with the Helsinki Declaration of 1975, as revised in 1983 and was approved by the Ethics Committee of Shengjing Hospital of China Medical University. Informed consent was obtained from the guardians of the study participants, and sample collection was performed in accordance with the standards of diagnosis and treatment.

\section{Study design}

Participants were assigned to groups according to a 2019 expert consensus [8] on the diagnosis and treatment of neonatal sepsis. In the sepsis group, $1 \mathrm{~mL}$ of venous blood was drawn to measure the IL-6 level when the infection was first clinically suspected (before the administration of antibiotics); 6 hours and 12 hours after infection onset; and 24-48 hours, 3 days, and 7 days after infection control. Another $2 \mathrm{~mL}$ of venous blood was drawn to measure the CRP and PCT levels at infection onset (before the administration of antibiotics) and 7 
days after infection control. Additionally, $1 \mathrm{~mL}$ of blood was drawn from the femoral vein for bacterial culture before the administration of antibiotics. In the control group, $3 \mathrm{~mL}$ of venous blood was drawn after admission for measurement of IL-6, CRP, and PCT. This study adheres to CONSORT guidelines.

\section{Index and methods}

IL-6 was measured by enzyme-linked immunosorbent assay with a reagent provided by Xiamen Huijia Biotechnology Co., Ltd. (Xiamen, China), and $\geq 96.52 \mathrm{pg} / \mathrm{mL}$ was considered positive [9]. CRP was measured by immune scattering turbidimetry with a reagent provided by Shanghai Jiemen Biotechnology Co., Ltd. (Shanghai, China), and $\geq 8 \mathrm{mg} / \mathrm{L}$ was considered positive. PCT was quantitatively measured by enzyme-linked fluorescence assay with a reagent produced by BRAHMS Co. (Hennigsdorf, Germany), and $\geq 0.5 \mathrm{ng} / \mathrm{mL}$ was considered positive.

\section{Definitions and diagnostic criteria}

A confirmed sepsis diagnosis was defined as clinical manifestations of sepsis and a positive blood or cerebrospinal fluid (or other sterile body cavity fluid) culture [8].

A clinical sepsis diagnosis was defined as clinical manifestations of sepsis with any of the following: more than two positive nonspecific blood detection indicators, cerebrospinal fluid showing signs of purulent meningitis; or the detection of pathogenic bacterial DNA in the blood [8].

A nosocomial infection was defined as an infection occurring $\geq 48$ hours after hospital admission, including infections occurring during hospitalization and those occurring after discharge, as a result of hospital exposure to pathogens [10]. 


\section{Statistical analysis}

SPSS 22.0 (IBM Corp., Armonk, NY, USA) was used for the statistical analysis of the data. Continuous data were expressed as means \pm standard deviations, and $t$-tests were used for comparisons between groups. Discrete data were expressed as frequencies, and chi-square tests were used for comparisons between groups. The best cutoff values of IL-6, CRP, and PCT were determined using receiver-operating characteristic (ROC) curve analysis. The sensitivity, specificity, positive predictive values (PPVs), negative predictive values (NPVs), and areas under the ROC curves (AUCs) of the three inflammatory marker levels were calculated singly and in combination. The correlation analysis used single-factor regression analysis, with the levels of the indicators as independent variables. A $P$ value of $<0.05$ indicated that the difference was statistically significant. This study was retrospectively registered at 2 September 2020 in the China Clinical Trials Registry (http://www.chictr.org.cn/listbycreater.aspx, reference number: ChiCTR2000037817).

\section{Results}

\section{Participant characteristics}

There were 89 cases of sepsis ( 47 cases of confirmed sepsis and 42 cases of clinical sepsis) and 31 controls (noninfected neonates hospitalized during the same period). There were seven cases of congenital heart disease, seven cases of neonatal encephalopathy, six cases of low birth weight, four cases of neonatal hyperbilirubinemia, two cases of neonatal anemia, two cases of gastrointestinal bleeding, and three cases of arrhythmia. 

statistically significant differences in gestational age, age, sex, or birth weight between the

144 neonates in the three groups.

\section{Blood culture}

A total of 47 neonates in the sepsis group had positive blood cultures, and the distribution of pathogens was as follows: 23 cases of gram-negative bacilli (48.9\%), 20 cases of gram-positive cocci $(42.5 \%)$, and four cases of fungi (8.6\%) (Table 2$)$.

\section{IL-6, CRP, and PCT levels}

Before antibiotic treatment, the levels of IL-6, CRP, and PCT in the sepsis group were significantly higher than those in the control group. After effective infection control (7 days), the levels of IL-6, CRP, and PCT in the sepsis group were significantly reduced (Table 3).

\section{IL-6 at each stage of infection}

Previous studies have clarified the dynamic trends of CRP and PCT [11]. The IL-6 levels peaked at 6 hours after the onset of infection and decreased to normal levels 24-48 hours after the infection was effectively controlled (Table 4, Figure 1).

\section{Comparison of the diagnostic values of IL-6, CRP, PCT, and combined testing}

The ROC curves (Figures 2 and 3) revealed that the best cutoff values of IL-6, CRP, and PCT were $>99.6 \mathrm{pg} / \mathrm{mL},>9.27 \mathrm{mg} / \mathrm{L}$, and $>2.33 \mathrm{ng} / \mathrm{mL}$, respectively. PCT had a high sensitivity, specificity, PPV, NPV, and AUC value, making it an ideal single index for the early identification of nosocomial infection in preterm infants. When combining PCT and IL-6 or using a combination of CRP, IL-6, and PCT for testing, the sensitivity and specificity values were over $85 \%$, and the predictive values were high (Table 5). 


\section{Prevalence of elevated levels of inflammatory markers}

Before antibiotic treatment, the prevalence of elevated levels of the three indicators was higher in the clinical sepsis group than in the confirmed sepsis group and the control group (Table 6).

\section{Discussion}

Cytokines are proteins or small molecular polypeptides secreted by cells. They are powerful mediators of inflammation and are essential for body defense, damage repair, and signal transduction. The detection of cytokines provides new possibilities for the diagnosis and treatment of neonatal sepsis. Among them, the proinflammatory cytokine IL-6 is currently a focus of research [12]. The IL-6 gene is located on human chromosome 7 and encodes a glycoprotein with multiple biological activities. IL-6 is a multi-effect proinflammatory factor. Under normal circumstances, the body's IL-6 content is extremely low $(<10 \mathrm{pg} / \mathrm{mL})$. After pathogens invade the body, lymphocytes, monocytes-macrophages, and fibroblasts are stimulated and a large amount of IL-6 is produced. The IL- 6 then stimulates the production of other inflammatory factors and acute phase response proteins [13]. Newborns with a gestational age less than 30 weeks or whose mothers used glucocorticoids before delivery have significantly reduced IL-6 levels [14]. In addition, IL-6 can also be markedly increased due to certain tumors, stress, autoimmune diseases, and transplant rejection. However, the above conditions are rare in the neonatal period, and only infections are relatively common. Therefore, when IL-6 is markedly increased in a newborn, it indicates an infection. Under the action of bacterial cell wall substances, such as endotoxin, IL-6 is quickly released, reaching a peak within a few hours: the more serious the infection, 
the greater the increase. The half-life of IL-6 is about 100 minutes, and it decreases significantly $24-48$ hours after the appearance of clinical signs, which means that it can be used for the effective diagnosis of bacterial infections and can be used to accurately judge the severity and prognosis of the infection [15-17]. Verboon et al. [18] found that $90 \%$ of children with enterovirus infections had normal levels of IL-6, indicating that IL-6 could be used as an important indicator to distinguish between bacterial and viral infections. Moreover, a prospective, randomized control study showed that IL-6 levels increase significantly 2 days before the onset of signs of bacterial infection, which is before other laboratory indicators indicated positive results [19]. The IL-6 level was negatively correlated with the time of blood collection, and the timing of its peak after infection was earlier than that of PCT. Combined with the finding that it had no physiological peak, the IL-6 level could be regarded as a sensitive indicator for the early diagnosis of neonatal sepsis. However, there are certain limitations to using IL-6 for guiding the empiric administration of antibiotics. IL-6 has a short half-life and peaks early. If the blood sample is not collected in time, the test may provide false-negative results. Moreover, the IL-6 level can return to normal while infection still persists [20]. Hatzidaki et al. [21] tested the IL-6 levels in cord blood, maternal blood during delivery, and serum on the fourth day after birth in 109 newborns who were born prematurely due to the premature rupture of membranes. The results showed that when the cutoff value of IL-6 was $108.5 \mathrm{pg} / \mathrm{mL}$, the sensitivity for diagnosis of sepsis was $95 \%$ and the specificity was $100 \%$, suggesting that IL-6 levels in the cord blood of neonates with a high risk of neonatal sepsis could help predict EOS. In this study, the best cutoff value of IL-6 was $99.6 \mathrm{pg} / \mathrm{mL}$. The results of our study were consistent with the results of a meta-analysis by Hu et al. [22]. 
The IL-6 level peaked at 6 hours after infection, which was much earlier than the levels of

209 CRP and PCT, and returned to normal 24-48 hours after the onset of infection and showed the highest sensitivity. Therefore, if preterm neonates have an elevated IL-6 level, physicians should be alerted to the possibility of a nosocomial infection, even if signs of infection are not present and levels of other inflammatory indicators are not abnormal, Natural immune cells can express Toll-like receptors or nucleotide-binding oligomerization domain-like receptors to identify various invading pathogens and release tumor necrosis factor alpha (TNF- $\alpha$ ) and proinflammatory factors, such as IL-1 $\beta$ and IL-6. Then, these proinflammatory factors promote the release of acute phase proteins (such as CRP and PCT). CRP is mainly synthesized in the liver. It is an acute response protein 218 produced by body tissues after various injuries or inflammatory stimuli and is mainly regulated by TNF- $\alpha$ and IL- 6 secreted by fat cells. CRP binds to the teichoic acid of Gram-positive bacteria and binds to the lipopolysaccharides of gram-negative bacteria. It is released 4-6 hours after the onset of infection, reaches its peak in 24-48 hours, and has a half-life of about 24 hours [23, 24]. CRP increases with the increase of inflammatory factors and decreases after infection control. It is positively correlated with the severity of infection. Therefore, a significant increase can be used as an early sign of bacterial sepsis. However, some non-infectious factors, such as neonatal asphyxia, ischemic or traumatic tissue damage, meconium aspiration syndrome, immunization, and hemolysis, could also cause an increase in CRP, resulting in low specificity of CRP as an indicator of sepsis [25]. Hengst et al. [26] reported that dynamic monitoring of the CRP trend could assist in the diagnosis of infectious diseases and in evaluating the clinical efficacy of antibiotics. Moreover, a retrospective 
analysis by Cui and Xue [11] showed that CRP peaked 24-48 hours after infection occurred and returned to normal 7 days after the infection was effectively controlled, indicating its dynamic trend is of great significance in guiding the rational administration of antibiotics and monitoring of clinical efficacy. In this study, the level of CRP before treatment in the sepsis group was significantly higher than that in the control group, indicating that CRP could be used for the diagnosis of neonatal sepsis. The ROC curve analysis found that the best cutoff value of CRP was $9.27 \mathrm{mg} / \mathrm{L}$. Compared with PCT and IL-6, CRP had lower sensitivity and specificity; therefore, it should not be used as an independent marker for the early diagnosis of nosocomial infection in preterm infants.

Serum PCT is a glycoprotein without hormone activity that is synthesized by liver cells and monocytes and is a precursor of calcitonin. In bacterial infections, the PCT level increases under the stimulation of endotoxin and proinflammatory factors (IL-6, TNF- $\alpha$, and IL-1ß). However, in viral infections, interferon gamma inhibits the synthesis and release of PCT [27]. PCT is not affected by factors such as maternal PCT level and stress and is only related to the extent of bacterial infection. The level of PCT in healthy people is extremely low $(<0.1 \mathrm{ng} / \mathrm{mL})$, and PCT is only secreted by thyroid neuroendocrine C cells. When the body is infected, it can be released from the cells of almost all tissues, and a level $\geq 0.5 \mathrm{ng} / \mathrm{mL}$ is defined as abnormal. A study by Lee et al. [28] showed that PCT started to increase 4 hours after the onset of infection and remained at the peak level for 8-24 hours. After control of the infection, the PCT level decreased by 50\% per day. However, Cui and Xue [11] found that PCT peaked 12 hours after the onset of infection and returned to normal within 3 days after infection control. This study found that in the sepsis group, the serum PCT level before the 
initiation of antibiotic treatment was significantly higher than that after treatment and was higher than the level in the control group, suggesting that the PCT level was positively correlated with the severity of infection. This is consistent with the results of a study by Canpolat et al. [29]. Auriti et al. [30] conducted a prospective, controlled study on 762 neonates from multiple centers. It was found that the serum PCT levels of neonates with sepsis were significantly higher than those of healthy neonates, with the best cutoff value of $2.4 \mathrm{ng} / \mathrm{mL}$, which had a sensitivity of $62 \%$ and specificity of $84 \%$. In this study, the best cutoff value of PCT was $2.33 \mathrm{ng} / \mathrm{mL}$, with a sensitivity of $84.0 \%$, specificity of $97.8 \%$. Therefore, serum PCT is of higher value for the early diagnosis of nosocomial infection in preterm infants, and the administration of antibiotics can be determined in the early stage of infection. Especially when the PCT level continues to remain high, it is necessary to consider replacing antibiotics. However, there is a physiological increase of PCT after birth. It is about $0.7 \mathrm{ng} / \mathrm{mL}$ at birth, peaks after 24 hours, and drops to below $0.1 \mathrm{ng} / \mathrm{mL}$ within 72 hours. This physiological pattern limits the diagnostic accuracy of serum PCT for the diagnosis of EOS [31]. Cord blood PCT has been reported to have good diagnostic accuracy for EOS, with a sensitivity of $82 \%$ and a specificity of $86 \%$ [32]. Moreover, a retrospective analysis by Joram et al. [33] showed that the average cord blood PCT level of neonates with EOS was 3.03 $\mathrm{ng} / \mathrm{mL}$, which was significantly higher than the $0.16 \mathrm{ng} / \mathrm{mL}$ of noninfected neonates. With the cutoff value of cord blood PCT as $0.6 \mathrm{ng} / \mathrm{mL}$, the NPV of PCT was 99\%, which made it a useful indicator for diagnosing EOS.

This study found that when the PCT result was combined with the IL-6 or with both IL-6 and CRP results, the sensitivity increased to $96.6 \%$ and $86.5 \%$, respectively; and the 
specificity increased to $87.1 \%$ and $96.8 \%$, respectively. In addition, the PPVs for PCT in combination with IL-6 or with both IL-6 and CRP were $98.3 \%$ and $98.2 \%$, respectively; the NPVs were $78.4 \%$ and $77.6 \%$, respectively; and the AUCs were 0.966 and 0.965 , respectively. These two combinations could both improve the accuracy of PCT for the early diagnosis of nosocomial infection in preterm infants.

The incidence of neonatal sepsis has steadily increased, and the prevalence of drug resistance has also increased. The incidence of methicillin-resistant, coagulase-negative Staphylococcus aureus and Escherichia coli has increased, and Acinetobacter baumannii and multidrug-resistant bacterial infections have been appearing in neonates. The results of this study showed that gram-negative bacteria were isolated significantly more frequently than gram-positive bacteria in the blood cultures of the neonates with sepsis. The three most common pathogenic bacteria were Klebsiella pneumoniae (34.0\%), Staphylococcus epidermidis (21.3\%), and Escherichia coli (12.8\%), showing that gram-negative bacteria are the main cause of neonatal sepsis and that Klebsiella pneumoniae was the dominant bacteria, which is consistent with the results of Wei [34].

This study also found that the prevalence of positive results of IL-6, CRP, and PCT in the confirmed sepsis group was lower than that in the clinical sepsis group. This might be due to false-negative results, insufficient blood samples, or test errors.

\section{Conclusion}

In summary, IL-6, CRP, and PCT could be used as reliable indicators for the early identification of nosocomial infections in preterm infants. IL-6 levels peaked 6 hours after the onset of infection, much earlier than those of CRP and PCT. Therefore, when IL-6 is elevated, 
296 physicians should be highly alert to the possibility of nosocomial infection in preterm infants.

297 The ROC curve analysis revealed that the best cutoff values of IL-6, CRP, and PCT

298 were $>99.60 \mathrm{pg} / \mathrm{mL},>9.27 \mathrm{mg} / \mathrm{L}$, and $>2.33 \mathrm{ng} / \mathrm{mL}$, respectively. In single-marker

299 measurements, the sensitivity, specificity, PPVs, NPVs, and AUC values of PCT were high,

300 making PCT an ideal index for the early identification of nosocomial infections in preterm

301 infants. Combined testing using PCT combined with IL-6 or with both CRP and IL- 6 could

302 improve the accuracy of clinical identification of nosocomial infections in preterm infants.

303 Testing these markers is fast and convenient and is conducive to early diagnosis and

304 treatment. Large sample sizes and multicenter studies are needed to confirm this result.

306 Abbreviations

307 AUCs: Areas under the ROC curves;

308 CI: Confidence interval;

309 CRP: C-reactive protein;

310 EOS: Early-onset sepsis;

311 IL-6: Interleukin 6;

312 LOS:Late-onset sepsis;

313 NPVs: Negative predictive values;

314 PCT: Procalcitonin;

315 PPVs: Positive predictive values;

316 ROC: Receiver-operating characteristic;

317 SD: Standard deviation. 


\section{Declarations}

Authors' contributions

320

CX and FJ: designed the research; $\mathrm{CX}, \mathrm{YH}$, and SY: conducted the research and

collected data; CX: wrote the manuscript; XX and SY: directed the writing of the manuscript;

FJ: had primary responsibility for final content. All authors designed, supervised, and

analyzed the study and assisted with the preparation of the manuscript. All authors read and

approved the final manuscript.

Funding

326

None.

Acknowledgements

Not Applicable.

\section{Availability of data and materials}

The data sets generated and analyzed during the current study are available in the

Harvard Dataverse repository (https://doi.org/10.7910/DVN/HD8MXB).

\section{Ethics approval and consent to participate}

The study was approved by the Medical Ethics Committee of Shengjing Hospital of

China Medical University.

\section{Consent for publication}

Informed consent was obtained from the infants' parents.

\section{Competing interests}

The authors declare that they have no competing interests. 


\section{Trial registration}

This study was retrospectively registered at 2 September 2020 in the China Clinical

341 Trials Registry (http://www.chictr.org.cn/listbycreater.aspx, reference number:

342 ChiCTR2000037817)..

\section{References}

344 1. Fleischmann-Struzek C, Goldfarb DM, Schlattmann P, Schlapbach LJ, Reinhart K,

345 Kissoon N. The global burden of paediatric and neonatal sepsis: a systematic review. The

346 Lancet Respiratory Medicine. 2018;6:223-30.

347 2. Wang H, Bhutta ZA, Coates MM, Coggeshall M, Dandona L, Diallo K, et al. Global, 348 regional, national, and selected subnational levels of stillbirths, neonatal, infant, and under-5

349 mortality, 1980-2015: a systematic analysis for the Global Burden of Disease Study 2015.

$350 \quad$ Lancet. 2016;388:1725-74.

351 3. Krajčinović SS, Doronjski A, Barišić N, Stojanović V. Risk factors for neonatal sepsis and 352 method for reduction of blood culture contamination. Malawi Med J. 2015;27:20-4.

4. Zea-Vera A, Ochoa TJ. Challenges in the diagnosis and management of neonatal sepsis. J Trop Pediatr. 2015;61:1-13.

5. Dong Y, Speer CP. Late-onset neonatal sepsis:Recent developments. Archives of Disease in Childhood: Fetal and Neonatal Edition. 2015;100:F257-63.

6. Liu L, Oza S, Hogan D, Perin J, Rudan I, Lawn JE, et al. Global, regional, and national causes of child mortality in 2000-13, with projections to inform post-2015 priorities: An updated systematic analysis. Lancet. 2015;385:430-40.

7. Hofer N, Müller W, Resch B. Non-infectious conditions and gestational age influence 
361 C-reactive protein values in newborns during the first 3 days of life. Clin Chem Lab Med. 2011;49:297-302.

8. The Subspecialty Group of Neonatology, the Society of Pediatric CMA. [Expert consensus on the diagnosis and management of neonatal sepsis (version 2019)]. Zhonghua er ke za zhi $=$ Chinese J Pediatr. 2019;57:252-7.

9. Procianoy RS, Silveira RC. The role of sample collection timing on interleukin-6 levels in early-onset neonatal sepsis. J Pediatr (Rio J). 2004;80:407-10.

10. Barbara J. Stoll. Infections of the neonatal infant. In: Kliegman RM, Behrman RE, Jenson HB, Stanton BMD, editors. Textbook of pediatrics. Philadelphia: WB Saunders; 2007. p. $794-811$.

11. Cui XW, Xue XD. Combined detection of procalcitonin and C-reactive protein in the early diagnosis of neonatal nosocomial infection. Chinese Pediatr Emerg Med. 2016;23:222-6. 
16. Oda S, Hirasawa H, Shiga H, Nakanishi K, Matsuda KI, Nakamua M. Sequential measurement of IL-6 blood levels in patients with systemic inflammatory response syndrome (SIRS)/sepsis. Cytokine. 2005;29:169-75.

17. Sharma D, Farahbakhsh N, Shastri S, Sharma P. Biomarkers for diagnosis of neonatal sepsis: a literature review. J Matern Neonatal Med. 2018;31:1646-59.

18. Verboon-Maciolek MA, Thijsen SFT, Hemels MAC, Menses M, Van Loon AM, Krediet TG, et al. Inflammatory mediators for the diagnosis and treatment of sepsis in early infancy. Pediatr Res. 2006;59:457-61.

19. Celik IH, Demirel FG, Uras N, Oguz SS, Erdeve O, Biyikli Z, et al. What are the cut-off levels for IL-6 and CRP in neonatal sepsis? J Clin Lab Anal. 2010;24:407-12.

20. Hou T, Huang D, Zeng R, Ye Z, Zhang Y. Accuracy of serum interleukin (IL)-6 in sepsis diagnosis: A systematic review and meta-analysis. Int J Clin Exp Med. 2015;8:15238-45.

21. Hatzidaki E, Gourgiotis D, Manoura A, Korakaki E, Bossios A, Galanakis E, et al. Interleukin-6 in preterm premature rupture of membranes as an indicator of neonatal outcome. Acta Obstet Gynecol Scand. 2005;84:632-8.

22. Hu J, Du PF, Bei DD. Diagnostic value of interleukin 6 for neonatal sepsis: A Meta analysis. Chinese J Contemp Pediatr. 2015;17:1176-82.

23. Stalenhoef JE, Van Nieuwkoop C, Wilson DC, Van Der Starre WE, Van Der Reijden TJK, Delfos NM, et al. Procalcitonin, mid-regional proadrenomedullin and C-reactive protein in predicting treatment outcome in community-acquired febrile urinary tract infection. BMC Infect Dis. 2019;19.

24. Haenssgen MJ, Charoenboon N, Althaus T, Greer RC, Intralawan D, Lubell Y. The social 
role of C-reactive protein point-of-care testing to guide antibiotic prescription in Northern Thailand. Soc Sci Med. 2018;202:1-12.

25. Shah BA, Padbury JF. Neonatal sepsis an old problem with new insights. Virulence. $2014 ; 5: 170-8$.

26. Hengst JM. The role of C-reactive protein in the evaluation and management of infants with suspected sepsis. Advances in Neonatal Care. 2003;3:3-13.

27. Meisner M. Pathobiochemistry and clinical use of procalcitonin. Clinica Chimica Acta. 2002;323:17-29.

28. Lee J, Bang YH, Lee EH, Choi BM, Hong YS. The influencing factors on procalcitonin values in newborns with noninfectious conditions during the first week of life. Korean $\mathrm{J}$ Pediatr. 2017;60:10-6.

29. Canpolat FE, Yiğit Ş, Korkmaz A, Yurdakök M, Tekinalp G. Procalcitonin versus CRP as an early indicator of fetal infection in preterm premature rupture of membranes. Turk J Pediatr. 2011;53:180-6.

30. Auriti C, Fiscarelli E, Ronchetti MP, Argentieri M, Marrocco G, Quondamcarlo A, et al. Procalcitonin in detecting neonatal nosocomial sepsis. Arch Dis Child Fetal Neonatal Ed. $2012 ; 97$

31. Chiesa C, Natale F, Pascone R, Osborn JF, Pacifico L, Bonci E, et al. C reactive protein and procalcitonin: Reference intervals for preterm and term newborns during the early neonatal period. Clin Chim Acta. 2011;412:1053-9.

32. Chauhan N, Tiwari S, Jain U. Potential biomarkers for effective screening of neonatal sepsis infections: An overview. Microbial Pathogenesis. 2017;107:234-42. 
427 33. Joram N, Muller JB, Denizot S, Orsonneau JL, Caillon J, Rozé JC, et al. Umbilical cord 428 blood procalcitonin level in early neonatal infections: A 4-year university hospital cohort 429 study. Eur J Clin Microbiol Infect Dis. 2011;30:1005-13.

430 34. Wei KL. The status and prospect of neonatal infection in China. Chinese J Pract Pediatr. $431 \quad 2011 ; 26: 1-2$. 
434 Figure 1. Mean interleukin 6 levels in the neonates with sepsis according to time since the 435 infection onset.

436 Figure 2. Receiver-operating characteristic curves for diagnosing neonatal sepsis according 437 to the mean IL-6, CRP, and PCT values. All tests were performed on admission to hospital.

438 Figure 3. Receiver-operating characteristic curves for diagnosing neonatal sepsis according 439 to the combinations of IL-6, CRP, and PCT values. All tests were performed on admission to 440 hospital. 
Table 1. Participant characteristics according to group

\begin{tabular}{cccc}
\hline Group & Confirmed sepsis group & Clinical sepsis group & Control group \\
\hline $\mathrm{N}$ & 47 & 42 & 31 \\
Sex (male/ female) & $25 / 22$ & $21 / 21$ & $20 / 11$ \\
Gestational age (weeks) & $33.55 \pm 3.02$ & $33.71 \pm 2.51$ & $33.66 \pm 2.33$ \\
Age (days) & $14.32 \pm 9.74$ & $13.50 \pm 8.92$ & $11.58 \pm 7.42$ \\
Birth weight $(\mathrm{g})$ & $1784 \pm 677.6$ & $1918 \pm 666.1$ & $1913 \pm 626.7$ \\
\hline
\end{tabular}


Table 2. Pathogens identified in neonates with sepsis

\begin{tabular}{lll}
\hline Pathogen classification & Cases (n) & Proportion of cases (\%) \\
\hline Gram-negative bacilli & 23 & 48.9 \\
Klebsiella pneumoniae & 16 & 34.0 \\
Escherichia Coli & 6 & 12.8 \\
Acinetobacter baumannii & 1 & 2.1 \\
Gram-positive cocci & 20 & 42.5 \\
Staphylococcus epidermidis & 10 & 21.3 \\
Staphylococcus aureus & 5 & 10.6 \\
Enterococcus faecium & 4 & 8.5 \\
Streptococcus agalactiae & 2 & 2.1 \\
Fungus & 4 & 8.6 \\
Candida albicans & & 4.3 \\
Candida parapsilosis & & \\
\hline
\end{tabular}


Table 3. Mean IL-6, CRP, and PCT levels according to group

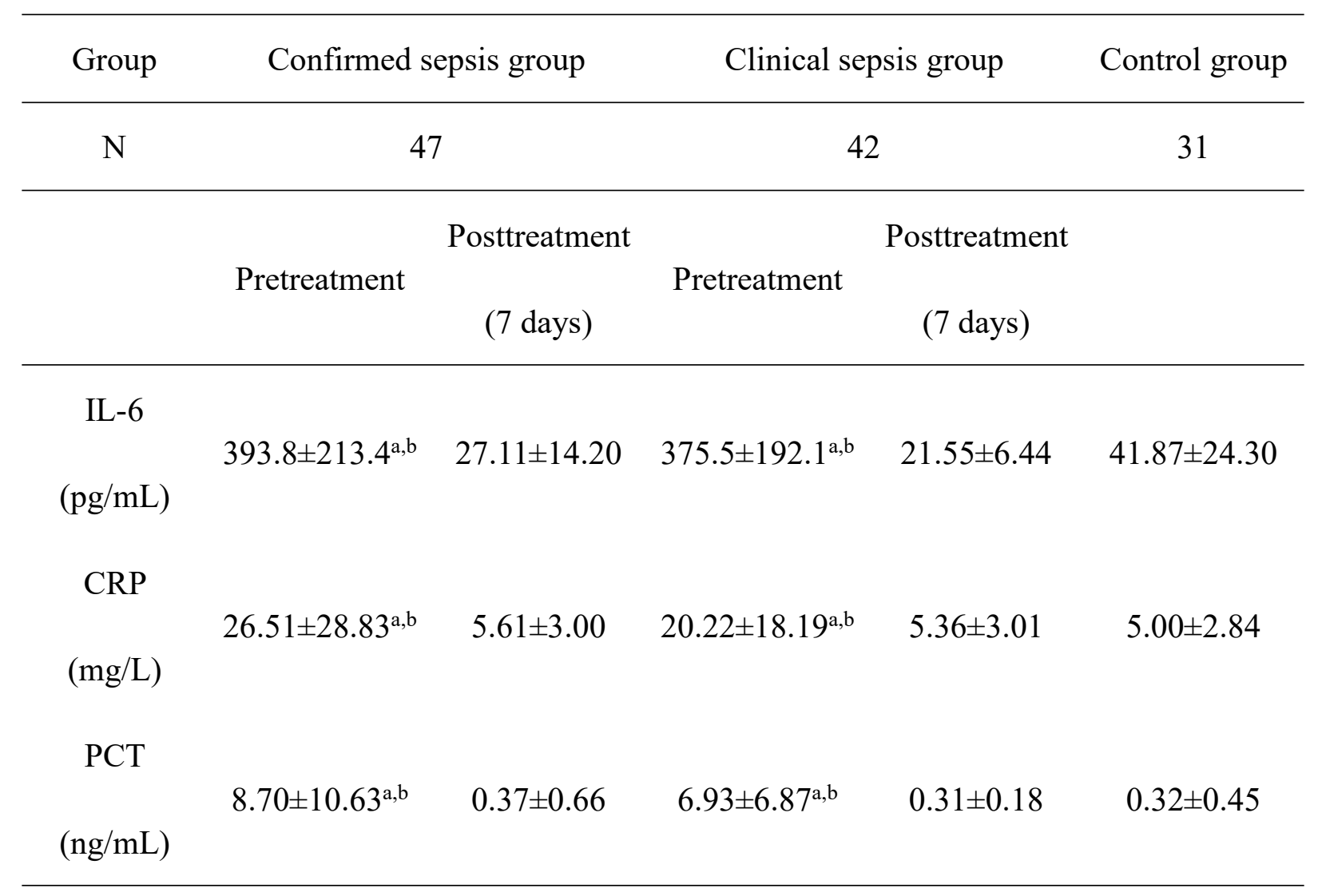

447 after treatment. 
Group

$$
\text { IL-6 (pg/mL) }
$$

$($ mean $\pm \mathrm{SD})$

\section{Sepsis group}

\section{Confirmed sepsis $(\mathrm{N}=47)$}

Pretreatment

$393.8 \pm 213.4$

Posttreatment (6 hours)

$1052 \pm 791.4$

Posttreatment (12 hours)

$270.6 \pm 160.8$

Posttreatment (24-48 hours)

$80.62 \pm 28.51$

Posttreatment (3 days)

$49.03 \pm 18.85$

Posttreatment (7 days)

$27.11 \pm 14.20$

\section{Clinical sepsis $(\mathrm{N}=42)$}

Pretreatment

$375.5 \pm 192.1$

Posttreatment (6 hours)

$877.2 \pm 431.4$

Posttreatment (12 hours)

$264.2 \pm 140.1$

Posttreatment (24-48 hours)

$67.95 \pm 16.89$

Posttreatment (3 days)

$37.49 \pm 9.40$

Posttreatment (7 days)

$21.55 \pm 6.44$ 
451 Table 5. The diagnostic accuracy of IL-6, CRP, and PCT, singly and in combination, for making the 452 diagnosis of sepsisa

\begin{tabular}{|c|c|c|c|c|c|c|c|}
\hline & & & $95 \% \mathrm{CI}$ & & & & \\
\hline Inflammatory marker & Cutoff value & AUC & of AUC & Sensitivity (\%) & Specificity (\%) & PPV (\%) & NPV (\%) \\
\hline IL-6 & 99.60 & 0.888 & $0.815-0.960$ & 87.6 & 91.3 & 96.3 & 71.8 \\
\hline CRP & 9.27 & 0.823 & $0.746-0.900$ & 62.9 & 87.1 & 93.3 & 45.0 \\
\hline PCT & 2.33 & 0.953 & $0.916-0.990$ & 84.0 & 97.8 & 98.3 & 78.4 \\
\hline $\mathrm{CRP}+\mathrm{PCT}$ & & 0.954 & $0.919-0.990$ & 91.0 & 83.9 & 98.2 & 77.7 \\
\hline $\mathrm{CRP}+\mathrm{IL}-6$ & & 0.939 & $0.893-0.985$ & 93.3 & 83.1 & 94.9 & 45.9 \\
\hline PCT+IL-6 & & 0.966 & $0.936-0.997$ & 96.6 & 87.1 & 98.3 & 78.4 \\
\hline $\mathrm{CRP}+\mathrm{PCT}+\mathrm{IL}-6$ & & 0.965 & 0.934-0.996 & 86.5 & 96.8 & 98.2 & 77.6 \\
\hline
\end{tabular}

${ }^{a}$ All tests were performed before the administration of antibiotics. 
454 Table 6 . The positive rates of IL-6, CRP, and PCT best cutoff values

\begin{tabular}{lccc}
\hline Group & IL-6 & CRP & PCT \\
\hline Sepsis group & & & \\
Confirmed sepsis group & 55.31 & 53.19 & 84.36 \\
Clinical sepsis group & 73.80 & 73.80 & 85.71 \\
Control group & 3.22 & 12.90 & 9.67 \\
\hline
\end{tabular}

455 


\section{Supplementary Files}

This is a list of supplementary files associated with this preprint. Click to download.

- CONSORT2010Checklist.doc 